\title{
Knee Point-Guided Multiobjective Optimization Algorithm for Microgrid Dynamic Energy Management
}

\author{
Wenhua Li $\mathbb{i},{ }^{1}$ Guo Zhang, ${ }^{1}$ Tao Zhang, ${ }^{1,2}$ and Shengjun Huang $\mathbb{D}^{1,2}$ \\ ${ }^{1}$ College of Systems Engineering, National University of Defense Technology, Changsha 410073, China \\ ${ }^{2}$ Hunan Key Laboratory of Multi-Energy System Intelligent Interconnection Technology, Changsha 410073, China
}

Correspondence should be addressed to Shengjun Huang; huangshengjun@nudt.edu.cn

Received 25 September 2020; Revised 16 October 2020; Accepted 21 October 2020; Published 7 November 2020

Academic Editor: Shi Cheng

Copyright (c) 2020 Wenhua Li et al. This is an open access article distributed under the Creative Commons Attribution License, which permits unrestricted use, distribution, and reproduction in any medium, provided the original work is properly cited.

Model predictive control (MPC) technology can effectively reduce the bad effect caused by inaccurate data prediction in microgrid energy management problem. However, the use of MPC technology needs to dynamically select an optimal solution from the Pareto solution set to implement, which needs the participant of the decision-makers frequently. In order to reduce the burden on decision-makers, we designed a knee point-based evolutionary multiobjective optimization algorithm, termed KBEMO. Knee point is the solution on Pareto front with the maximum marginal utility, which is considered as the preferred solution if there is no other preference. This algorithm focuses on obtaining the knee region and automatically outputs knee points after the optimization. By combining this algorithm with MPC technology, it can effectively reduce the amount of computational consumption and obtain better convergence. Experimental results show that this method is more competitive than the traditional singleobjective MPC method.

\section{Introduction}

In recent years, energy shortages and environmental pollution problems have become increasingly severe. In order to improve the safety and survivability of traditional power grid, microgrid technology has received more and more attention, especially in absorbing large-scale wind and solar energy into the power grid, which has great application prospects. The concept of microgrid was first proposed by the American Electric Power Technology Research Institute, the Consortium for Electric Reliability Technology Solutions (CERT) [1]. It is a small power supply and distribution autonomous system that contains a variety of microgrid sources, e.g., electrical loads, energy storage devices, and energy conversion devices. The microgrid can be self-regulated, monitored, and self-protected [2, 3].

The uncertainty of renewable energy in the microgrid increases the risk of system scheduling. How to adapt to the uncertain working conditions of various renewable energy output and realize the benign interaction of multiple resources and economic operation is a difficult task of the research in energy management and optimization [4]. The main problem in energy management is how to formulate a power generation plan and dispatch plan with the lowest overall operating cost under the stable operation of the system in response to the uncertainty in the process of power generation and power consumption so as to meet the needs of production and life better. With the development of microgrid technology, more and more energy supply and energy-consuming equipment are connected to the microgrid. However, renewable energy power generations such as wind and solar power and users' energy demand are random and uncertain. Existing studies have shown that the method of day-ahead scheduling [5] is useful. Specifically, energy management optimization is performed based on the forecast results of the next 24 hours. However, the result often differs from the actual situation. In response to this problem, Zhang et al. [6] introduced a model predictive control (MPC) framework to optimize the microgrid energy management problem. The main process can be summarized as follows: (1) in each period, the microgrid system will predict the renewable energy power generation and user 
load of the next 24 hours; (2) optimize the operation based on the predicted data; (3) apply the optimal solution obtained at the current moment and run the system according to real-world data; (4) update the status of the microgrid system; and (5) return to the first step and repeat the whole process. The MPC framework [7] continuously optimizes the energy management problem during the continuous advancement of the period, thereby reducing the impact of uncertain factors. This method has been successfully applied to many microgrid energy management problems, and a high-quality solution has been achieved.

Microgrid energy management is a typical multiobjective optimization problem (MOP). We need to consider optimizing more than one objective when dealing with the microgrid energy management problem, e.g., the loss of energy storage system, stability of power supply, energy-saving, and pollutant emission reduction [8]. To deal with MOPs, the traditional linear programming method generally adopts the method of weighted sum (WS); that is, the decision-maker gives a set of weight vectors in advance and then couples the objective vector and the weight vector into a scalar function for optimization. Although this method is simple to apply, it has many problems. On the one hand, due to the inconsistency of the scales between different objective functions, it is difficult to determine the exact value of the weight vector, resulting in poor robustness when optimizing the single-objective optimization problem. Specifically, the use of larger weights will increase the influence of the objective function noise, leading to unilateral preference. On the other hand, it is often difficult for decision-makers to give his appropriate preference weights in advance.

To address the issue of using the WS method to solve MOP, evolutionary multiobjective optimization (EMO) has attracted more and more attention $[9,10]$. The EMO algorithm can give a satisfactory solution set when the number of objective functions is 2 or 3 and has been successfully applied to solve a large number of real-world engineering problems [11]. Without loss of generality, the definition of a multiobjective optimization problem is as follows:

$$
\begin{aligned}
\text { Minimize, } F(\mathbf{x}) & =\left\{f_{1}(\mathbf{x}), f_{2}(\mathbf{x}), \ldots, f_{m}(\mathbf{x})\right\}, \\
\text { s.t., } \quad \mathbf{x} & =\left(x_{1}, x_{2}, \ldots, x_{n}\right) \in \Omega,
\end{aligned}
$$

where $\Omega$ denotes the feasible decision space, $m$ is the number of objectives, and $\mathbf{x}$ is a decision vector consisting of $n$ decision variables $x_{i}$. A solution $\mathbf{x}_{\mathbf{a}}$ is said to Pareto dominate another solution $\mathbf{x}_{\mathbf{b}}$ if and only if $\forall i=1,2, \ldots, m, f_{i}\left(\mathbf{x}_{\mathbf{a}}\right) \leq f_{i}\left(\mathbf{x}_{\mathbf{b}}\right)$ and $\exists j=1,2, \ldots, m, f_{j}\left(\mathbf{x}_{\mathbf{a}}\right)<f_{j}\left(\mathbf{x}_{\mathbf{b}}\right)$. The images of all Pareto optimal solution sets (PSs) in the objective space are termed the Pareto optimal front (PF).

Generally speaking, the EMO algorithm will give a set of Pareto optimal solution that does not dominate each other and no solution can outperform other solutions on all objective functions. Since it is always necessary to select a solution from many Pareto optimal solutions to apply in real-world engineering problems, general multiobjective optimization problems need to be combined with corresponding decision-making methods. When the number of goals is small, such as 2-3 goals, the decision-makers can intuitively select the appropriate solution from the Pareto front according to their own preferences by plotting all solutions. However, it is pointed out from cognitive studies that when the number of objectives is greater than 5, the applicability of this type of method is greatly reduced. In response to this problem, researchers have proposed many posterior methods, such as grey correlation analysis [12] and TOPSIS method [13].

Microgrid energy management combined with the MPC framework is a dynamic optimization problem. To solve this problem by the EMO algorithms, it is necessary to use a posteriori method to select an optimal solution for application after optimization, which will lead to the waste of computational resource. Generally speaking, the decision-maker is only interested in a small area of Pareto front, and other solutions on the Pareto front will waste a lot of computing resources. Therefore, focusing on certain representative areas of the Pareto front is of great significance for solving real-world engineering problems. Research points out that knee, as the point with the greatest marginal utility on the Pareto front, is more attractive to decision-makers [14]. For the two-objective optimization problem, the knee in the Pareto front refers to the solution with the largest marginal rate of return; that is, a small improvement in one objective will lead to serious degradation of at least another objective [15], as shown in Figure 1. Intuitively, the knee area is a "bulge" on the front of Pareto, where the slope changes suddenly. Many works have discussed the importance of knee and pointed out that, for general MOPs, if the decision-maker has no other preference, the knee can be used as the preferred solution.

Based on the above considerations, to solve the online microgrid energy management problem by the EMO algorithm, we first proposed a microgrid energy management mathematical model combined with the MPC framework. Moreover, a knee-based EMO algorithm (KBEMO) is proposed to solve the energy management problem, which can focus on obtaining the knee region on the Pareto front and give the global knee point as the final solution. Combined with this algorithm, the optimal solution is adopted continuously after each optimization process. Therefore, the microgrid can run as time goes, and the impact of renewable energy and user load uncertainty in the microgrid can be reduced. As a result, the running cost of the microgrid can be improved. Experiments show that this method can effectively obtain the optimal result of the microgrid energy management problem and reduce the impact of randomness.

The rest of this paper is organized as follows: Section 2 introduces the related models of microgrid energy management; Section 3 describes the knee-based EMO algorithm in detail and its application under the MPC framework; the experimental settings and experimental results are mentioned in Section 4; finally, we summarize the advantages and disadvantages of the current method and propose possible future research directions. 


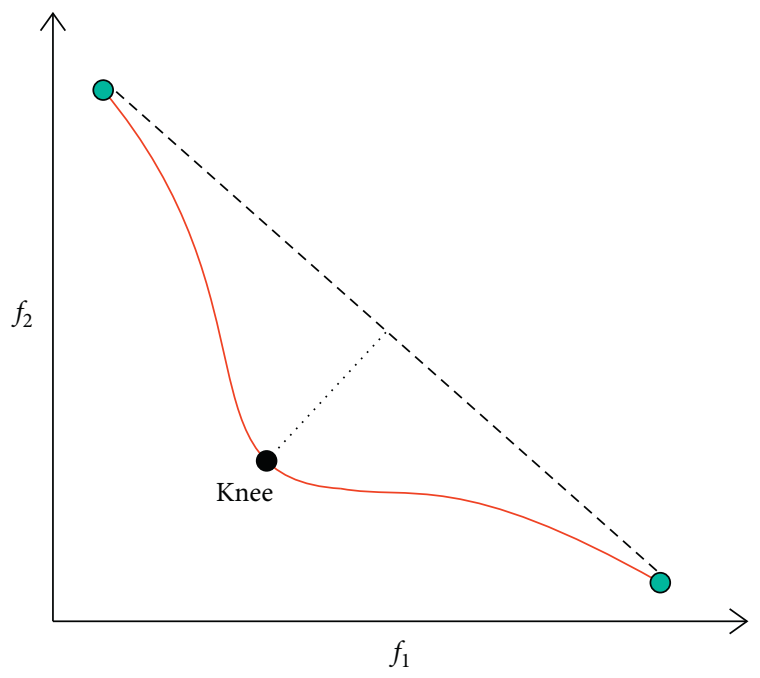

Edge point

Pareto front

FIGURE 1: Illustration of knee point.

\section{Microgrid Energy Management Model}

Since this paper mainly discusses the application of the EMO algorithm in dynamic microgrid energy management, we select a typical microgrid model, which specifically includes renewable energy (wind and solar), energy storage system, user load, and the power grid. The time-ofuse electricity price strategy is adopted, which means that the electricity price changes over time. Therefore, by introducing an energy storage system, we can purchase electricity from the power grid when the electricity price is low and sell electricity to the power grid when the price is high. Therefore, the overall operating cost of the microgrid can be reduced. Since there are many parameters involved in this model, the relevant parameters are summarized as follows (Table 1).

With the development of microgrid technology, more and more energy supply and energy-consuming equipment are introduced, and the model of microgrid energy management has become more and more complex. This article mainly studies the application of knee-based algorithms in dynamic energy management, so it considers the basic microgrid composition, including renewable energy power generation, energy storage systems, user loads, and large power grids.

As one of the most flexible and directly controllable devices in the microgrid, the energy storage system can not only be used as a consumer device to absorb excess electric energy but also as a backup power supply when the power generation is insufficient. Its constraints mainly include the maximum and minimum capacity limits and charge and discharge energy transfer power, which can be expressed as

$$
\begin{aligned}
& P_{\text {bess }}^{\min } \leq P_{\text {bess }}(k) \leq P_{\text {bess }}^{\max }, \\
& E_{\text {bess }}^{\min } \leq E_{\text {bess }}(k) \leq E_{\text {bess }}^{\max },
\end{aligned}
$$

$$
E_{\text {bess }}(k+1)=E_{\text {bess }}(k)+\eta_{\text {bess }} P_{\text {bess }}(k) \Delta t .
$$

Equations (2)-(4), respectively, represent the charge and discharge power constraints, energy level constraints, and dynamic energy transfer constraints of the energy storage system. Energy storage systems have different battery losses under the different state of charge (SOC), which can be expressed as

$$
f_{\text {soc }}(k)=1-\frac{1}{1+\alpha^{*} \exp \left(\beta^{*} \operatorname{soc}(k)\right)} .
$$

Among them, $\operatorname{soc}(k)=E(k) / E_{\text {bess }}^{\max }$ represents the current state of the energy storage system and $\alpha$ and $\beta$ characterize the internal parameters of the energy storage system, which is provided by the manufacturer. Similarly, the interaction between the microgrid system and the power grid also needs to meet the upper and lower power limits:

$$
P_{\text {grid }}^{\min } \leq P_{\text {grid }}(k) \leq P_{\text {grid }}^{\max } .
$$

The prerequisite for the economic dispatch of the microgrid is that the power supply and consumption of the microgrid system are always balanced, which is expressed as

$$
P_{\text {solar }}(k)+P_{\text {grid }}(k)=P_{\text {bess }}(k)+P_{\text {load }}(k) \text {. }
$$

Many objective functions can be considered for microgrid energy management problems. We take the economic operation of the microgrid and the loss of the energy storage system as the optimization objectives, which can be expressed as

$$
\begin{aligned}
& \min f_{1}=\sum C_{\text {grid }}(k)=\sum c_{\text {grid }}(k) P_{\text {grid }}(k) \Delta t, \\
& \min f_{2}=\sum C_{\text {bess }}(k)=\sum f_{\text {soc }}(k) .
\end{aligned}
$$

Among them, $f_{1}$ is the cost incurred by the interaction between the microgrid and the power grid and $f_{2}$ represents the loss of the energy storage system incurred during the operation.

The day-ahead scheduling technology of the microgrid means that, for the period that needs to be scheduled, the forecasting method is used to obtain data such as future renewable energy generation and user load. According to the data, the microgrid energy management is optimized, and the operation plan in the future period is obtained. In actual operation, it is difficult to accurately predict the data, so it will be adjusted according to the real-time data and the principle of power balance to achieve the purpose of stable operation.

\section{Knee-Based Multiobjective Optimization Algorithm}

3.1. Motivation and Framework. Generally speaking, the process of the algorithm based on the knee can be summarized as detecting the knee on the Pareto front and guiding the evolutionary search direction according to the knee information, so as to obtain the knee area. In recent years, researchers have proposed many methods on how to 
TABLe 1: Parameter name and meaning.

\begin{tabular}{lcc}
\hline Parameter type & Symbol & Meaning \\
\hline & $T$ & Control period of the model \\
System parameters & $P_{\text {grid }}^{\min } P_{\text {grid }}^{\max }$ & $E_{\text {burs }}^{\text {cur }}$ \\
& $E_{\text {bess }}^{\max } E_{\text {bess }}^{\min }$ & Upper and lower power limits of interaction with the power grid \\
& $P_{\text {bess }}^{\min } P_{\text {bess }}^{\max }$ & Initial energy storage level of the energy storage system \\
& $\varepsilon_{\text {bess }}$ & Upper and lower limits of energy storage system capacity \\
& $\eta_{\text {bess }}$ & Upper and lower limits of charging power for energy storage system \\
Predictive variable & $P_{\text {solar }}(k)$ & Self-discharge energy loss of energy storage system \\
& $P_{\text {load }}(k)$ & Energy storage system charging and discharging efficiency \\
Decision variable & $\delta_{\text {bess }}^{c}(k)$ & Power of PV prediction generation \\
& $P_{\text {bess }}^{c}(k)$ & Power of load prediction generation \\
Cost & $C_{\text {bess }}(k)$ & Energy storage system charge and discharge status \\
& $C_{\text {grid }}(k)$ & Energy storage system charge and discharge power
\end{tabular}

detect knee. Das [16] proposed a knee point detection method based on the normal boundary intersection (NBI). Das defines an extreme line for the two-objective problem, called individual minimal convex hull (CHIM), which passes through the two boundary points (also called edge points) of the Pareto front. As shown in Figure 1, the knee point corresponds to the solution furthest from CHIM. This method is also known as a distance-based knee detection method and has been successfully applied to algorithms such as KnEA [14] and k-NSGA-II [9].

The existing knee detection methods can be mainly divided into two categories: (1) detection based on the geometric characteristics of the Pareto front. Unlike other parts of the Pareto front, the knee area has obvious geometric characteristics, that is, a curvature of the Pareto front. Representative methods include distance-based method [16], minimum Manhattan distance [17] (MMD), and reflection angle [15]. (2) Use Pareto front to evaluate the tradeoffs between solutions. In the knee region, the influence between the objective function values increases. The knee region is detected by calculating such influences. Representative methods include cone-domination [18], trade-off [19], and expected marginal utility [15] (EMU) method. The performance of each method in knee detection is compared in [20] in detail, which pointed out that the distance-based detection method has strong robustness. However, the distance-based method is highly sensitive to the position of the boundary point, and different algorithms have different determinations of the Pareto front boundary. Therefore, this method may cause the problem of inaccurate knee detection.

The minimum Manhattan distance (MMD) method calculates the Manhattan distance of each solution on the Pareto front, and the solution with the minimum Manhattan distance is the current global knee point. The calculation method of this method is simple and has strong robustness. Therefore, we adopt the MMD method to detect the knee. The specific steps are as follows:

(1) Standardize the objective function values of the currently obtained solutions $x^{(1)}, \ldots, x^{(k)}$ :

$$
f_{m}^{\prime}\left(x^{(i)}\right)=\frac{f_{m}\left(x^{(i)}\right)-z_{m}^{*}}{z_{m}^{\text {nad }}-z_{m}^{*}},
$$

where $z^{*}$ and $z^{\text {nad }}$ are the best and worst values of all Pareto optimal solutions and $f_{m}$ and $f_{m}^{\prime}$ are the original target value and the normalized target value of the $m$-th dimension, respectively.

(2) Calculate the Manhattan distance of the solution $x^{(i)}$ on the Pareto front:

$$
f_{1}^{\prime}\left(x^{(i)}\right)+f_{2}^{\prime}\left(x^{(i)}\right)+\cdots+f_{M}^{\prime}\left(x^{(i)}\right)=v
$$

(3) The solution with the smallest Manhattan distance is the knee point of the current evolutionary process.

It can be seen from Figure 2 that the Manhattan distance of the solution $x^{(i)}$ can be expressed as a straight line with a slope of -1 , which passes through the solution $x^{(i)}$ and the intercept is $v$. This paper detects the knee based on the MMD method. After obtaining the current knee information, the information is used to guide the evolution direction of the population, and the final knee region is obtained based on the NSGA-II [21] algorithm. The algorithm framework is illustrated in Algorithm 1.

After the algorithm detects the knee, it calculates the environmental fitness value of all individuals based on the knee information (line 8 ). The calculation process can be expressed as follows:

(1) Calculate the crowding distance of all individuals $x^{(i)}$ according to the crowding distance $\mathrm{cd}^{(i)}$ calculation method of the NSGA-II algorithm.

(2) If the Euclidean distance $D_{i, j}$ between the individual $x^{(i)}$ and the knee point in the objective space is less than the given threshold $\delta$, the crowding distance of solution $x^{(i)}$ will be rewarded according to the following equation: 


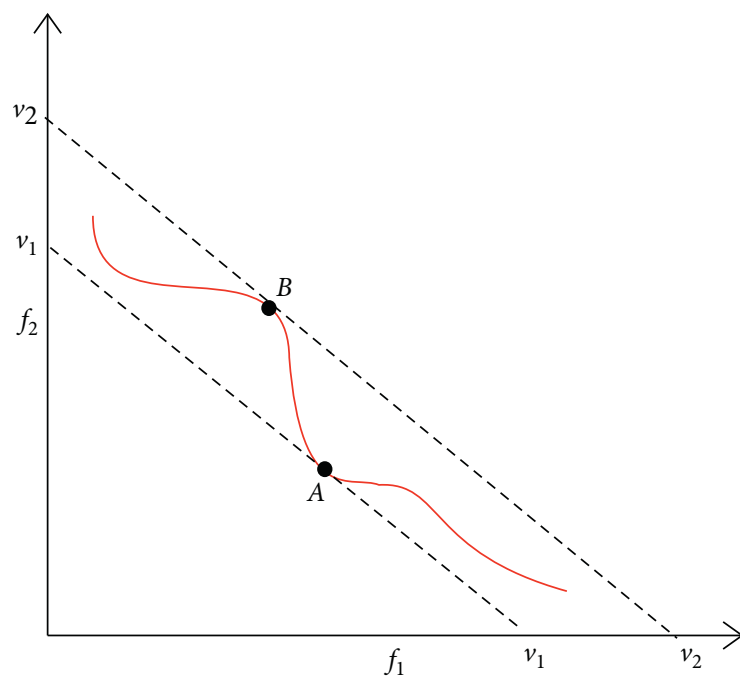

FIgURE 2: Illustration of the MMD method.

$$
\mathrm{cd}^{(i)}=\mathrm{cd}^{(i)} \cdot\left(1+\frac{1}{1+\exp \left(D_{i, j}-\delta\right)}\right) .
$$

Among them, $1 / 1+\exp \left(D_{i, j}-\delta\right)$ is the activation function; that is, when $D_{i, j}<\delta, \mathrm{cd}^{(i)}$ will be rewarded. The threshold $\delta$ is set by the user and is used to adjust the size of the knee area finally obtained.

Since the NSGA-II algorithm is likely to choose solutions with a larger crowding distance to form the next generation during the evolution, by increasing the crowding distance of individuals that are near to the current knee, the fitness value of such individuals is improved. In the process of evolution, the solutions around the knee are retained. On the other hand, by detecting the knee point during the evolution process, we can utilize the knee as the current elite individual. Moreover, the excellent genes of current knee solution will be shared with other solutions through crossover (line 5) to improve the convergence performance of the algorithm. It is worth noting that the edge point on the Pareto front of the multiobjective optimization problem plays a guiding role in obtaining the Pareto front. Besides, in some special situations, the decision-maker is interested in the solution of the boundary point, so the proposed algorithm treats the boundary point as knee points as well so that the boundary points can be retained during the evolution process.

\subsection{Dynamic Optimization of Microgrid Energy Management.} The day-ahead scheduling method predicted data for some time in the future (usually a day) to obtain the running strategy in advance. In actual running time, it will dynamically adjust based on actual data to satisfy the energy balance constraints. Although this method can make a reasonable control strategy for system operation to a certain extent, it has many disadvantages. Because it is difficult to accurately predict the renewable energy generation and user loads, in the actual operation process, the operating state of the system is also different from that of the preplanning stage. Applying solutions obtained based on inaccurate forecast data will make the actual running cost more expensive than the plan.

Model predictive control [19] will plan the operation strategy for a period of time in the future based on the predicting data and execute the obtained operation strategy at the current moment. At the next moment, the system state and the prediction for the future will be updated based on actual operating data, and the new planning operation strategy is applied at this moment. By continuously repeating the above process, the microgrid energy management system continues to obtain the operating strategy of the microgrid. Because this method continuously adjusts the optimizer based on actual data, it can reduce the impact of randomness and reduce the requirements for prediction accuracy.

After the EMO algorithm obtains the Pareto solution set, it needs to introduce the preference of the decision-maker to choose a solution for implementation. However, in dynamic optimization problems, it is unrealistic to frequently require decision-makers to participate, so we must find a way to automatically select a solution that satisfies the preference of decision-maker. The knee point on the Pareto front is the most "cost-effective" point in the entire solution set, which is regarded as the better solution when there is no other preference. In the model predictive control framework, the current global knee is selected as the optimal solution for implementation so that decision-makers do not need to provide preference information. It is worth noting that when there are multiple knees in the Pareto front of the problem, the algorithm will select the global knee as the optimal solution. The mainframe of the proposed method is shown in Figure 3.

It is worth noting that the optimization strategy of the current period has guiding significance for the next stage of optimization. In particular, when the forecast data are 100\% accurate, the microgrid energy management strategy derived at the current moment should be also suitable for the next stage. In order to make better use of the optimization information obtained in the previous period and reduce the computational waste, we select solutions from the final population in the current stage to form up the elite individuals, which is combined with the initial population for the next stage. By doing this, the optimizer can converge to the Pareto optimal front faster. Specifically, the boundary points and the points around the knee point are chosen. The reason to select only a small part of the population is that it is easy to fall into the local optimal solution if we directly use the current population as the initial population. For the excellent individuals in the previous stage, the coded sequence 2 to $T$ needs to be moved to the sequence 1 to $T-1$, and the value is randomly generated to fill the code $T$.

3.3. Experimental Parameter Setting. This paper proposed a microgrid energy management model including energy storage system, renewable energy power generation, user load, and power grid and selects a microgrid project as a case to verify the optimization method proposed in the 


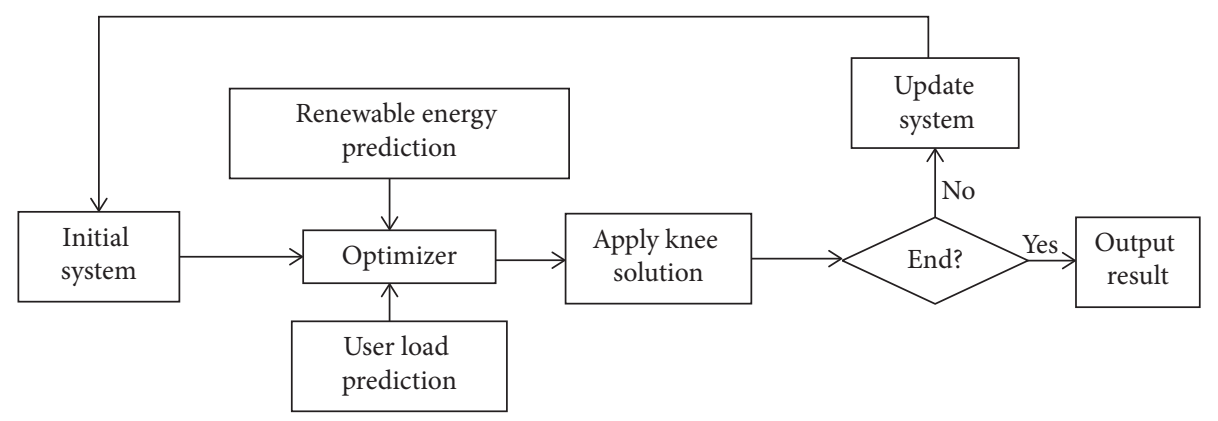

FIgURE 3: MPC framework optimization flow chart.

article. The main parameters of this microgrid are shown in Table 2. The relevant parameters of the knee-based EMO algorithm (hereinafter referred to as KBEMO algorithm) are shown in Table 3. For renewable energy power generation and user load data, we use the global energy forecasting competition 2014 data set and get the time-ofuse electricity price based on the local electricity price, as shown in Figure 4.

\section{Result and Analysis}

4.1. Performance of the Proposed Algorithm. In order to exam the performance of the proposed KBEMO algorithm in searching and obtaining the knee area, we selected the classic knee test problems to test the KBEMO algorithm. Specifically, Branke et al. [15] proposed DEB2DK, DEB3DK, and DO2DK problems based on the DTLZ test problem [22], and the parameter $\mathrm{K}$ is introduced to control the number of knees on the Pareto front. We independently run 31 times for each test problem, calculate the generation distance (GD) of the results obtained in each run, and select the result closest to the average GD for display.

Figure 5 shows the Pareto fronts obtained by the KBEMO algorithm on DEB2DK, DEB3DK, and DO2DK problems. It can be seen from the figure that KBEMO can accurately detect and obtain the knee area on the Pareto front, and the boundary points of the Pareto front can be completely preserved. In terms of algorithm convergence, KBEMO can be very close to the true Pareto front. Therefore, the KBEMO algorithm can completely find and retain the knee area on the selected test problem, and it has great convergence performance.

4.2. Algorithm Performance Comparison. In order to compare the difference between the KBEMO algorithm and the classic multiobjective optimization algorithm, we compare the Pareto fronts obtained by KBEMO and MOEA/D-DE for the same problem. We choose MOEA/D-DE [23] to represent the classic multiobjective optimization algorithm, which has been proven to have strong competitiveness in dealing with multiobjective optimization problems. To ensure fairness, the two algorithms use the same operating parameters and run 31 times independently and compare the results that best represents their average level. The results are shown in Figure 6.
It can be seen from Figure 6 that the Pareto front obtained by the KBEMO algorithm is closer to the theoretical optimal value than the classic optimization algorithm MOEA/D-DE, which shows that the KBEMO algorithm can obtain a better convergence solution under the same conditions. At the same time, the algorithm accurately gives the global knee point for this problem. The solution represented by this point can well balance the two objective functions, which can better represent the preference of the decisionmaker. For the traditional EMO algorithm, generally speaking, a larger population is required to describe the complete Pareto front, which greatly increases the waste of irrelevant calculations because the decision-maker does not need this information. Because the KBEMO algorithm only focuses on a specific area on the Pareto front, the required population is smaller and more advantageous, and because it focuses more on the search in a specific area, the algorithm has a stronger convergence ability than the normal algorithm.

4.3. Comparison of MPC Framework and Day-Ahead Scheduling. The method based on day-ahead scheduling (hereinafter referred to as DA) only needs to use the optimizer for a single-time optimization, so it has a great advantage in computational efficiency. However, the scheduling method obtained by this method is based on the premise of an accurate prediction of future data and the actual situation is also different from the forecast data. To compare the difference between the day-ahead scheduling and the MPC-based framework, we conduct energy management for the microgrid in the next 24 hours. In actual operation, we will design fluctuations in renewable energy generation and user load demand, and there is a $10 \%$ error between the prediction and the actual situation. In order to reduce the impact of randomness, all experiments are independently run for 31 times, and the averaged results are listed in Table 4.

It is worth noting that the optimization results based on the MPC framework are significantly better than the optimization results of the day-ahead scheduling. This is because when optimizing the energy management of the microgrid based on the MPC framework, the algorithm can update the system's status according to the actual operating data in each period. On the contrary, since there is no real-time feedback mechanism in the day-ahead scheduling method, its optimal 
Input: maximum generations Max Gen, population size $N$ Output: nondominated set PF, knee point $k c$

(1) $k c \longleftarrow \phi$ and $n \_n c \longleftarrow 0$

(2) PS $\longleftarrow$ Initialization $(N)$

(3) Costs $\longleftarrow$ Cal Obj Value (PS)

(4) While $i t_{\text {cur }} \leq i t_{\max }$

(5) OS $_{1} \longleftarrow$ Crossover (PS, $k c$ )

(6) $\mathrm{OS}_{2} \longleftarrow$ Mutation(PS)

(7) joint $S={\mathrm{PS} \cup \mathrm{OS}_{1} \cup \mathrm{OS}_{2}}_{2}$

(8) $\mathrm{EF} \longleftarrow$ Cal Env Fitness (joint $S, k c$ )

(9) joint $S \longleftarrow$ Non dominated Sort (joint $S$ )

(10) $\quad$ PS $\longleftarrow$ Env Selection (EF, joint $S$ )

(11) $k c \longleftarrow$ Knee Detection(PS)

(12) $i t_{\text {cur }}=i t_{\text {cur }}+1$

(13) End While

Algorithm 1: General framework.

TABle 2: Parameter values of the microgrid system in this research.

\begin{tabular}{lcccccccc}
\hline$T$ & $k$ & $\Delta t$ & $P_{\text {grid }}^{\min } P_{\text {grid }}^{\max }$ & $E_{\text {bess }}^{\text {cur }}$ & $E_{\text {bess }}^{\min } E_{\text {bess }}^{\max }$ & $P_{\text {bess }}^{\min } P_{\text {bess }}^{\max }$ & $\varepsilon_{\text {bess }}$ & $\eta_{\text {bess }}$ \\
\hline 24 hours & $12,18,24$ hours & 1 hour & $0,1000 \mathrm{~kW}$ & $500 \mathrm{kWh}$ & $0,2000 \mathrm{kWh}$ & $-200,200 \mathrm{~kW}$ & $0.2 \mathrm{kWh}$ & $95 \%$ \\
\hline
\end{tabular}

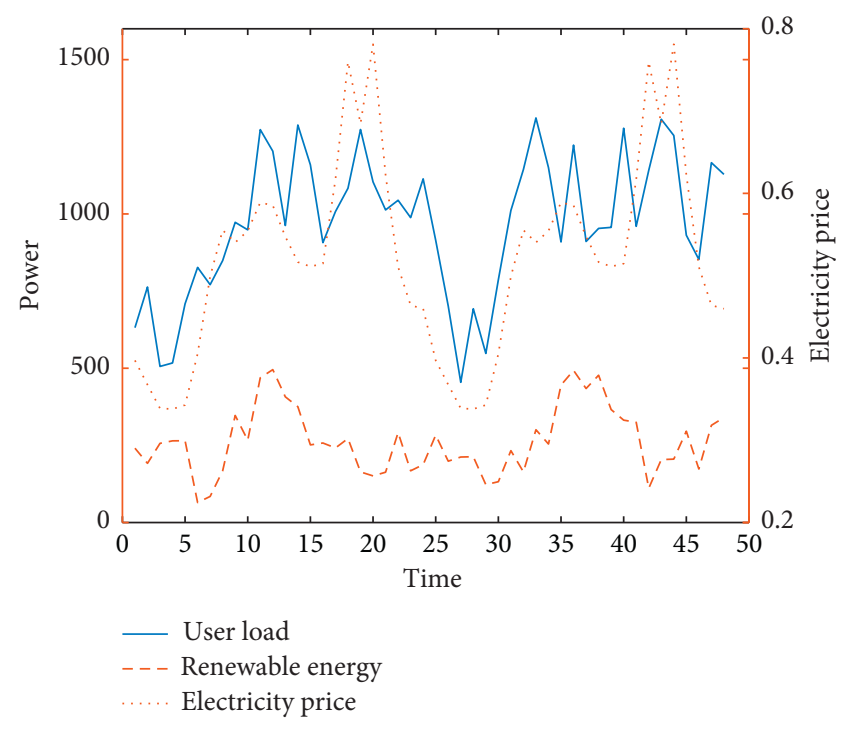

FIgURE 4: Schematic diagram of microgrid user load, renewable energy generation, and time-of-use electricity price.

results are poor. It is foreseeable that as the optimization period increases, the data prediction errors generated by the day-ahead scheduling will gradually accumulate, resulting in the continuous deterioration of the optimization effect.

4.4. Comparison of Results of Different Algorithms. The KBEMO algorithm proposed in this paper can utilize knee as the optimal solution for application after the optimization in each stage, without the need for decision-makers to give preference information. For the traditional method, the decision-maker needs to give a set of weight vectors in advance, after each optimization, and use the weighted sum
(WS) method to convert the objective function vector into a scalar value, thereby selecting the best solution. To compare the efficiency and performance of the WS method and the KBEMO method, we use the knee obtained by the KBEMO algorithm and the optimal solution obtained by the WS method under the MPC framework to solve the dynamic microgrid energy management problem. For the WS method, since the dimension of the objective function involved in this problem is inconsistent, after each run, the objective function value is first normalized. Since it is impossible to determine which weight can obtain the best performance, we set up multiple different weight vectors. In order to reduce the impact of randomness, all experiments were run 31 times independently, and the average of the results was taken as the final result.

Figure 7 shows the results that are closest to the average running result based on different weight vectors and the KBEMO algorithm. This result shows the running state of the charging and discharging power of the energy storage system and the interaction power with the power grid over time. It can be seen from the figure that both methods can make corresponding adjustments according to the time-ofuse electricity price. When the electricity price is relatively high, the optimizer chooses to use the energy storage system for discharge to reduce the cost of purchasing electricity from the power grid; on the contrary, when the electricity price level is low, the optimizer chooses to charge the energy storage system with high power and purchase electricity from the power grid. This shows that the proposed microgrid energy management model can well describe the operating status of the microgrid.

It is worth mentioning that the running state of the knee solution is a good trade-off of the two objective functions. Solutions with $w=[0.1,0.9]$ mainly consider to optimize 


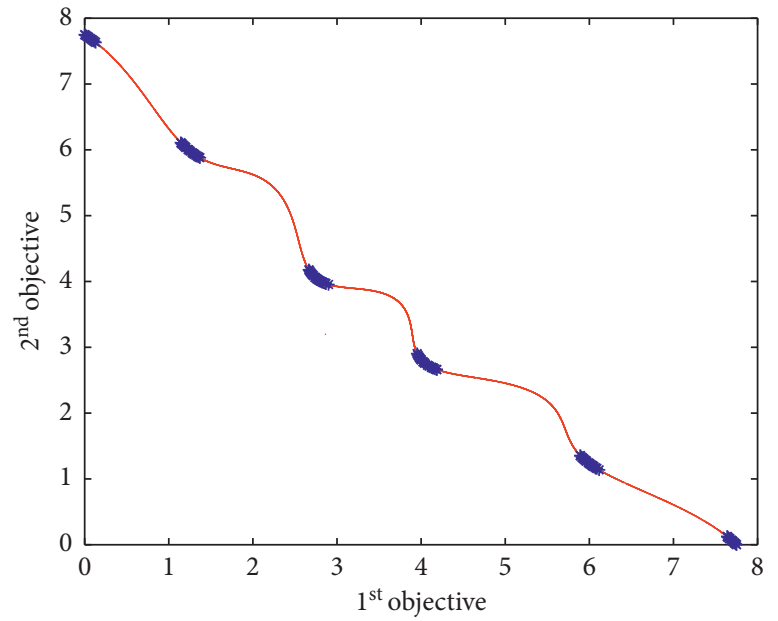

True Pareto front

* KBEMO’s Pareto front

(a)

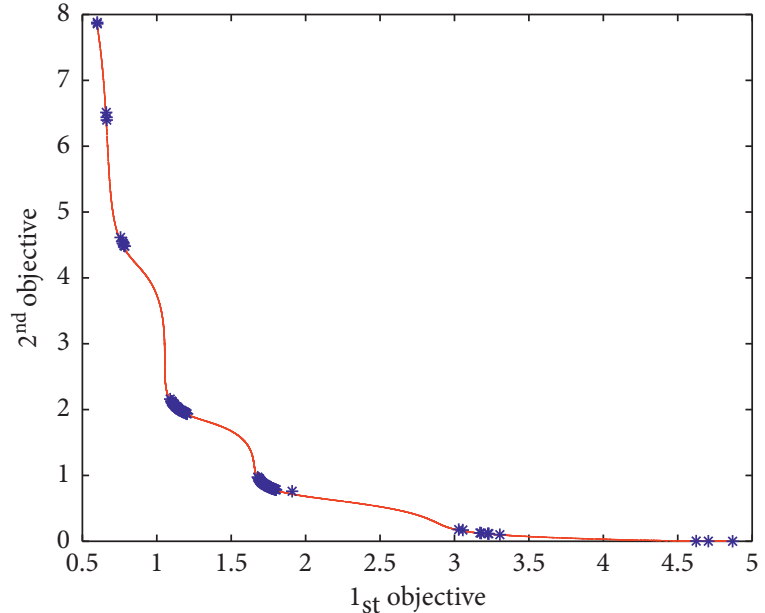

True Pareto front

* KBEMO's Pareto front

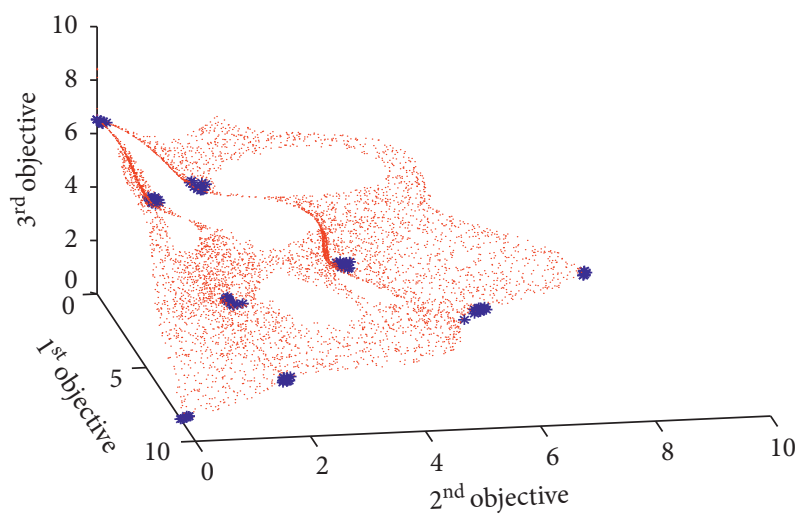

True Pareto front

* KBEMO's Pareto front

(c)

Figure 5: Pareto fronts obtained by KBEMO on knee test problems: (a) DEB2DK; (b) DO2DK; (c) DEB3DK.

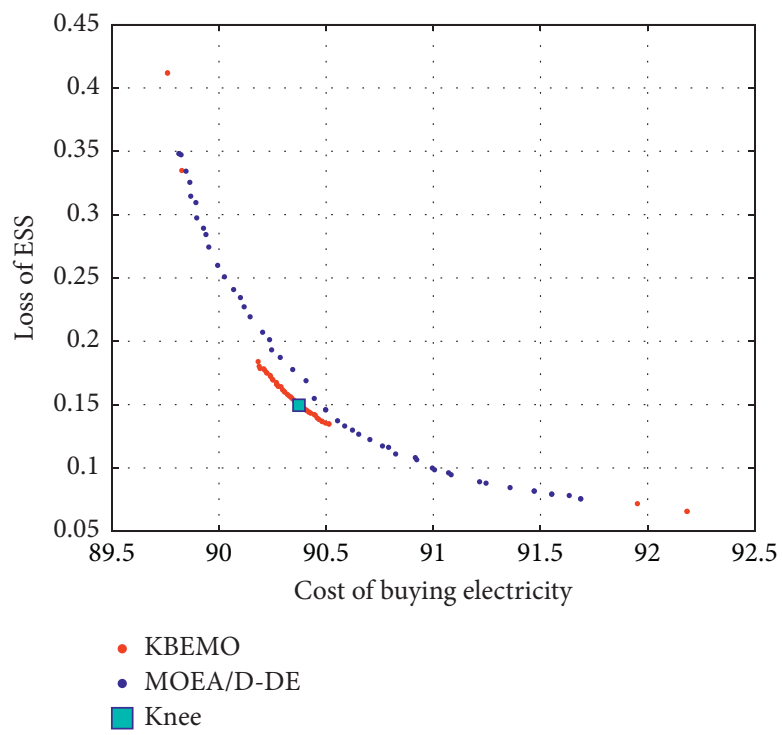

Figure 6: Pareto fronts obtained by MOEA/D-DE and KBEMO. 
TABLE 3: Algorithm running parameters.

\begin{tabular}{lcccc}
\hline The iterations of one time & Iterations & Cross probability & Mutation probability & Population size \\
\hline 100 & 40 & 0.7 & 0.3 & 80 \\
\hline
\end{tabular}

TABLE 4: Results of microgrid.

\begin{tabular}{lcc}
\hline Method & Actual cost of buying electricity & Loss of the energy storage system \\
\hline DA & $92.7634 \$$ & 0.7856 \\
MPC & $89.6854 \$$ & 0.5598 \\
\hline
\end{tabular}

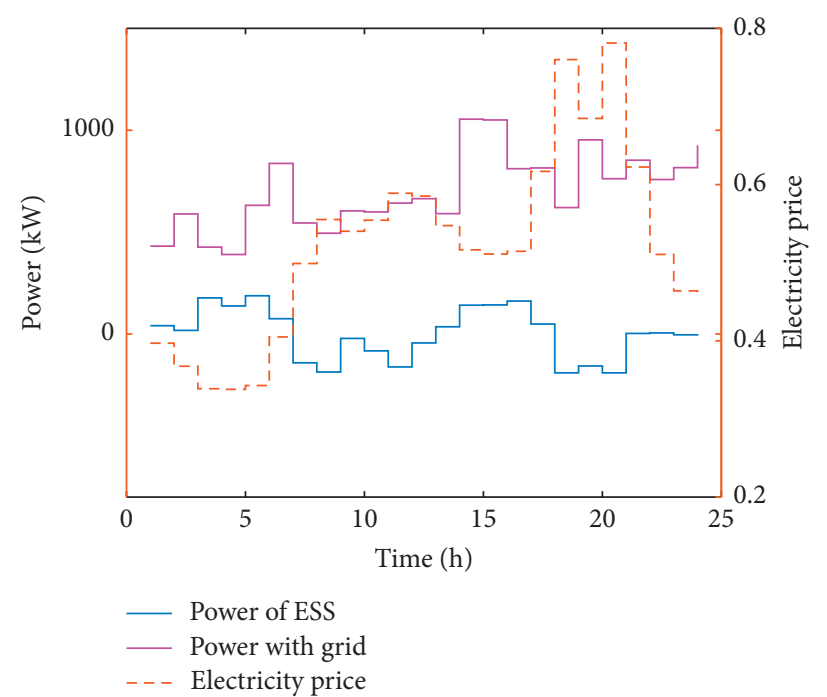

(a)

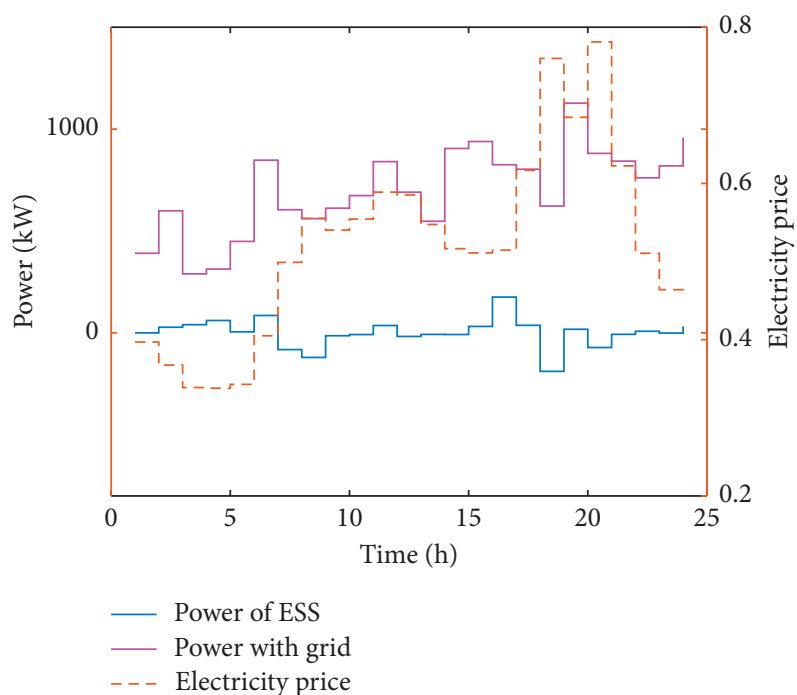

(b)

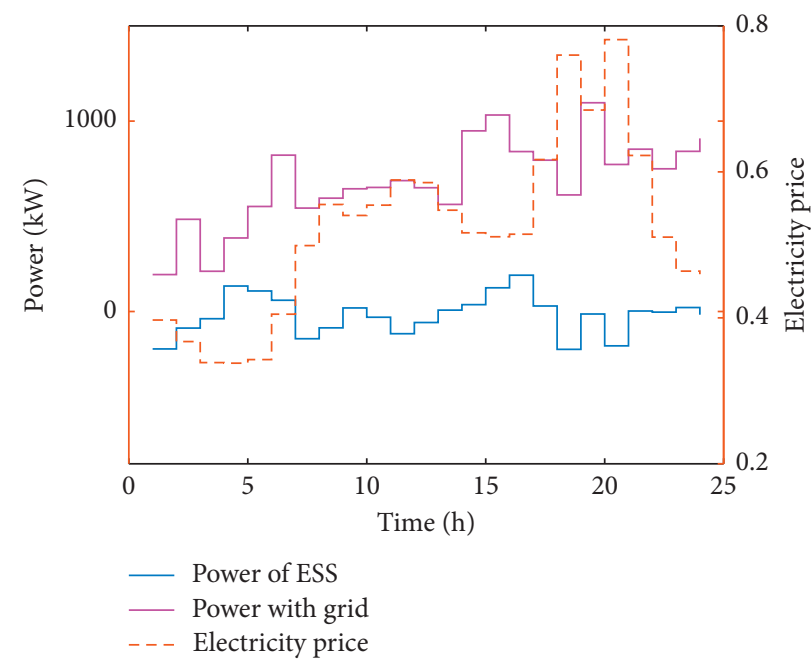

(c)

Figure 7: Operation states of different components: (a) $w=[0.1,0.9]$; (b) $w=[0.9,0.1]$; (c) knee.

the $2^{\text {nd }}$ objective function, and the loss of energy storage system is good but results in high electricity buying cost. On the contrary, the solution with $w=[0.9,0.1]$ will consider the $1^{\text {st }}$ objective function more.

It can be seen from Figure 8 that choosing solutions by different weights at each stage will result in the final result being more biased towards the objective function. Therefore, the WS method can reflect the preference information of the decision-maker to a certain extent. However, the results of most weight vectors under the WS method are inferior to the KBEMO algorithm. In other words, the objective function value obtained by the KBEMO algorithm dominates the objective function value obtained by the WS method under most weight values. On the one hand, during the operation 


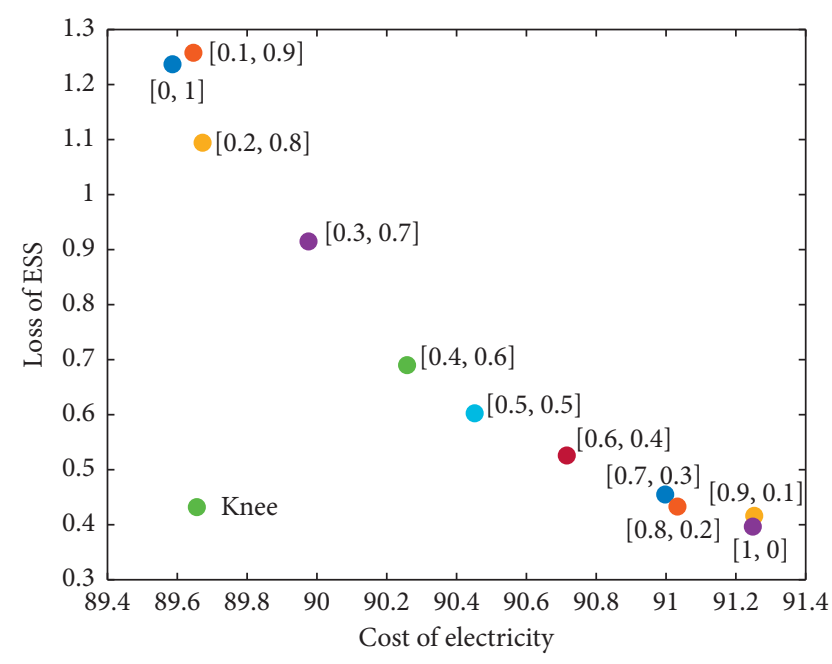

FIGURE 8: Solutions distribution of WS and KBEMO.

of the MPC framework, the KBEMO algorithm can obtain the most balanced solution every time, so the final solution obtained by this method can well balance the two objectives. The WS method selects the solution with a specific weight vector every time, and the trade-off between the two goals is poor, so the quality of the obtained solution is not high. On the other hand, because the $\mathrm{KB}$ algorithm focuses on searching the knee area, its convergence performance is better than the classic multiobjective optimization algorithm.

\section{Conclusion}

Microgrid plays an important role in making use of renewable energy and restraining fluctuation of the power grid supply. With the increase in the dimensions of the problem to be considered, the role of multiobjective optimization algorithms in the energy management of microgrids is becoming more and more important. Although the multiobjective optimization algorithm can give the Pareto optimal front of the problem, the decision-maker still needs to choose one solution from many solutions to apply. This will put pressure on decision-makers and consume computing resources to search for areas that decision-makers may not be interested in. Knee, as the largest marginal utility point on the Pareto front, is considered to be a more popular solution for decision-makers.

Aiming at the situation that the optimal solution needs to be continuously determined in the model predictive control, this paper proposes an evolutionary multiobjective optimization algorithm based on the knee, which can find the knee region of the Pareto front during the evolution process. The algorithm is used to optimize the energy management problem of the microgrid, and the knee is applied as the optimal solution in each period. Experiments have proved that this method can effectively obtain an optimized scheme for microgrid energy management and requires less computing resources.

Future research work includes the following: (1) fully considering the uncertainty of power generation and consumption and improving the multiobjective energy management method of microgrid combined with a model predictive control method. (2) Improving the knee-based EMO algorithm so that the algorithm can obtain the knee area of the problem more accurately and reliably and improve the computational efficiency of the algorithm. For now, the KBEMO is based on NSGA-II, which is proposed several years ago. Some recent algorithms with better performance can be considered [24-26]. (3) Consider applying the knee-based EMO algorithm to the multi-microgrid group, which provides new ideas for the efficient solution of the multi-microgrid system.

\section{Data Availability}

All data are included in the paper.

\section{Conflicts of Interest}

The authors declare that they have no conflicts of interest.

\section{Acknowledgments}

This work was supported by the National Natural Science Foundation of China (61773390), the Hunan Youth Elite Program (2018RS3081), the scientific key research project of National University of Defense Technology (ZK18-02-09 and ZZKY-ZX-11-04), and the Key Project of 193-A11-10103-01.

\section{References}

[1] R. Lasseter and P. Paigi, "Microgrid: a conceptual solution," in Proceedings of the 2004 IEEE 35th Annual Power Electronics Specialists Conference (IEEE Cat. No. 04CH37551), Aachen, Germany, June 2004.

[2] F. Wang, J. L. Duarte, and M. A. M. Hendrix, "Grid-interfacing converter systems with enhanced voltage quality for microgrid application-concept and implementation," IEEE Transactions on Power Electronics, vol. 26, no. 12, pp. 35013513, 2011.

[3] M. Sechilariu, B. Wang, and F. Locment, "Building-integrated microgrid: advanced local energy management for forthcoming smart power grid communication," Energy and Buildings, vol. 59, pp. 236-243, 2013.

[4] W. Li, R. Wang, T. Zhang, M. Ming, and H. Lei, "Multiscenario microgrid optimization using an evolutionary multiobjective algorithm," Swarm and Evolutionary Computation, vol. 50, Article ID 100570, 2019.

[5] G. Liu, Y. Xu, and K. Tomsovic, "Bidding strategy for microgrid in day-ahead market based on hybrid stochastic/ robust optimization," IEEE Transactions on Smart Grid, vol. 7, no. 1, pp. 227-237, 2015.

[6] Y. Zhang, L. Fu, W. Zhu, X. Bao, and C. Liu, "Robust model predictive control for optimal energy management of island microgrids with uncertainties," Energy, vol. 164, pp. 12291241, 2018.

[7] S. J. Qin and T. A. Badgwell, "A survey of industrial model predictive control technology," Control Engineering Practice, vol. 11, no. 7, pp. 733-764, 2003.

[8] S. Phrakonkham, J.-Y. Le Chenadec, D. Diallo, G. Remy, and C. Marchand, "Reviews on micro-grid configuration and 
dedicated hybrid system optimization software tools: application to Laos," Engineering Journal, vol. 14, no. 3, pp. 15-34, 2010.

[9] W. Li, R. Wang, T. Zhang, M. Ming, and K. Li, "Reinvestigation of evolutionary many-objective optimization: focus on the pareto knee front," Information Sciences, vol. 522, pp. 193-213, 2020.

[10] K. Li, R. Wang, T. Zhang, and H. Ishibuchi, "Evolutionary many-objective optimization: a comparative study of the state-of-the-art," IEEE Access, vol. 6, pp. 26194-26214, 2018.

[11] W. Li, T. Zhang, R. Wang, B. Wang, Y. Song, and X. Li, "A knee-point driven multi-objective evolutionary algorithm for flexible job shop scheduling," in Proceedings of the 2019 IEEE Symposium Series on Computational Intelligence (SSCI), pp. 1716-1722, Xiamen, China, December 2019.

[12] Y. Kuo, T. Yang, and G.-W. Huang, "The use of grey relational analysis in solving multiple attribute decision-making problems," Computers \& Industrial Engineering, vol. 55, no. 1, pp. 80-93, 2008.

[13] M. Behzadian, S. Khanmohammadi Otaghsara, M. Yazdani, and J. Ignatius, "A state-of the-art survey of TOPSIS applications," Expert Systems with Applications, vol. 39, no. 17, pp. 13051-13069, 2012.

[14] X. Zhang, Y. Tian, and Y. Jin, "A knee point-driven evolutionary algorithm for many-objective optimization," IEEE Transactions on Evolutionary Computation, vol. 19, no. 6, pp. 761-776, 2015.

[15] J. Branke, K. Deb, H. Dierolf, and M. Osswald, "Finding knees in multi-objective optimization," in Proceedings of the International Conference on Parallel Problem Solving from Nature, pp. 722-731, Birmingham, UK, September 2004.

[16] I. Das, "On characterizing the "knee" of the Pareto curve based on normal-boundary intersection," Structural Optimization, vol. 18, no. 2-3, pp. 107-115, 1999.

[17] W.-Y. Chiu, G. G. Yen, and T.-K. Juan, "Minimum Manhattan distance approach to multiple criteria decision making in multiobjective optimization problems," IEEE Transactions on Evolutionary Computation, vol. 20, no. 6, pp. 972-985, 2016.

[18] G. Yu, Y. Jin, and M. Olhofer, "An a priori knee identification multi-objective evolutionary algorithm based on $\alpha$-dominance," in Proceedings of the Genetic and Evolutionary Computation Conference Companion, pp. 241-242, Prague, Czech Republic, July 2019.

[19] L. Rachmawati and D. Srinivasan, "Multiobjective evolutionary algorithm with controllable focus on the knees of the Pareto front," IEEE Transactions on Evolutionary Computation, vol. 13, no. 4, pp. 810-824, 2009.

[20] G. Yu, Y. Jin, and M. Olhofer, "Benchmark problems and performance indicators for search of knee points in multiobjective optimization," IEEE Transactions on Cybernetics, vol. 50, no. 8, pp. 3531-3544, 2020.

[21] K. Deb, A. Pratap, S. Agarwal, and T. Meyarivan, "A fast and elitist multiobjective genetic algorithm: NSGA-II," IEEE Transactions on Evolutionary Computation, vol. 6, no. 2, pp. 182-197, 2002.

[22] K. Deb, L. Thiele, M. Laumanns, and E. Zitzler, "Scalable test problems for evolutionary multiobjective optimization," in Evolutionary Multiobjective Optimization, pp. 105-145, Springer, Berlin, Germany, 2006.

[23] H. Li and Q. Zhang, "Multiobjective optimization problems with complicated Pareto sets, MOEA/D and NSGA-II," IEEE Transactions on Evolutionary Computation, vol. 13, no. 2, pp. 284-302, 2009.
[24] R. Wang, R. C. Purshouse, and P. J. Fleming, "Preferenceinspired coevolutionary algorithms for many-objective optimization," IEEE Transactions on Evolutionary Computation, vol. 17, no. 4, pp. 474-494, 2013.

[25] R. Wang, Z. Zhou, H. Ishibuchi, T. Liao, and T. Zhang, "Localized weighted sum method for many-objective optimization," IEEE Transactions on Evolutionary Computation, vol. 22, no. 1, pp. 3-18, 2018.

[26] R. Wang, Q. Zhang, and T. Zhang, "Decomposition-based algorithms using Pareto adaptive scalarizing methods," IEEE Transactions on Evolutionary Computation, vol. 20, no. 6, pp. 821-837, 2016. 